the duration of these losses reduces them in proportion. To obtain economy in heat, therefore, the ideal is not only to insulate as well as practicable, but also to heat and cast the metal in as short a time as possible, and this ideal may be approached by having each $1 \mathrm{~b}$. of metal heated for the shortest possible time. The total loss of heat per $l b$. of metal while it is hot is the criterion. From this point of view Dr. Hering states that the ideally perfect melting furnace, if such it can be called, is the electric fuse, in which the intended result is completed in such an exceedingly short time - a fraction of a second-that the heat losses during that time are vanishingly small, and hence the therma efficiency is practically Ioo per cent.

With fuel heating, too great a rapidity of heating generally involves high chimney losses, i.e. a lower efficiency in heat transmission to the metal, and hence a limit to the speed is soon reached; but with electric heating there is no chimney loss, and the possibilities of rapid heating are therefore more encouraging. Electric arc heating involves high radiation losses from the arc itself, but in heating the metal by its own resistance the heat can be generated below the surface and in the metal itself, thereby eliminating al heat transmission losses. Extremely rapid heating then becomes possible, being limited only by the size of the heat-generating capacity provided, and in the case of brass or zinc by the volatilisation of the zinc in the part in which the heat is set free. By the resistance method, therefore, the ideal represented by the electric fuse can be approached more closely than by any other known method. Small high-speed furnaces are therefore, from this point of view, an approach to the ideal, particularly as they involve the minimum of contamination of the metal being melted. In Dr. Hering's opinion, it will in time become possible, for light castings at least, to be melted in an electric furnace about as fast as the metal can be cast, in which case the furnace would need to have a metal capacity of only enough for about two moulds. In that case it would be so small that it could be transported to the moulds, thereby saving the usual large heat losses in the transporting crucibles, besides the heat losses in the crucibles themselves.

Another factor, however, is involved, viz. the larger the amount of metal in a furnace, the less the rate of heat loss per Ib., because the larger the volume, the less is the surface exposed. In a large furnace with a hemispherical hearth the heat loss per $1 \mathrm{~b}$. of metal through walls having uniform insulation is reduced to about one-half when the capacity is increased from $\mathrm{I}$ to ro tons. Hence, for this reason, the larger the furnace the better.

In choosing between these two apparently conflicting ideals the following considerations must be borne in mind:-(i) When melting is the only object, then the metal should be kept hot the shortest possible time; hence there should be used as small a furnace as is consistent with the amount of metal required for one casting. (ii) When there are involved operations such as refining, mixing, uniformity of alloying, the taking of specimens for analysis while melted, or any other process requiring time, the larger the furnace the better.

\section{LIQUID FUEL.}

"I IQUID Fuel and its Combustion" was the title $\mathcal{L}$ of a paper read by Prof. J. S. S. Brame, on February 20, before the Institution of Petroleum Technologists. Attention was directed to the increasing use of liquid fuel, and especially to its connection with those developments of the internal combustion engine which have so largely determined the progress of aviation and submarine navigation. Nevertheless he recalls the warning of Redwood (1905) that no oil supplies are in sight sufficient to replace anything like the bulk of solid fuel consumed. The use of liquid fuel for steam raising and industrial heating is the special subject of the paper, and the following considerations are brought forward. In constancy of chemical composition, whatever the source, and therefore of calorific value, mineral fuel oils compare very favourably with coal, and accordingly physical considerations such as low viscosity and freedom from grit may decide the choice of oil fuels. Turning to our home supplies, it is gratifying to note that the heavy fractions of the Scotch shale oils are ideal in this respect; having been distilled they are clean, while their fluidity is very satisfactory. Another home product, which is deserving of the close attention of liquid fuel experts, is coal-tar, the supply of which must increase with the extension of coal carbonisation. Its production may outgrow its uses in normal channels, and as a home-made liquid fuel its rational utilisation is a matter of high national importance. Nevertheless, for marine purposes tar (and tar oils) must remain inferior to petroleum, since a higher oxygen content and lower calorific value are inevitable, while a capacity for giving off disagreeable fumes may make it objectionable in the confined space of a stokehold. Methods of burning oil are surveyed historically, leading up to the spray burners now almost invariably used which "atomise" the oil.

The method of spraying is varied, depending on the use of compressed air or steam, or on forcing oil alone under pressure through a suitable burner, a method specially adapted for use in marine boilers. On theoretical grounds air injection would seem to be most generally efficient; steam may propel oil satisfactorily into the fire, but afterwards its influence on combustion can only be of negative value. The general arrangements of the system for combustion have more bearing on the success of a plant than the choice of atomiser. It is too often overlooked that, compared with solid fuel, where burning is mainly confined to the fuel bed, oils require a much greater volume of combustion space.

Looking to the future, Prof. Brame points out how much depends on the development of the internal combustion engine; for naval purposes he believes that oil firing with turbines will hold the field.

J. W. C.

RECENT PROGRESS IN SPECTROSCOPY." II.

$R$ ADIATION is an electromagnetic process, $R$ and must be determined by the electrical state of the radiator. A molecule may be neutral or for a moment charged by the loss or gain of an electron. This type of ionisation must actually occur, as indicated by the conduction of electricity through the vapour of a compound which shows no evidence of chemical dissociation. What causes the light emission? It may accompany the loss or gain of an electron by a neutral molecule, in which case the emission centre would be charged. It may be due to the shock of elastic collision with an electron or ion, or to the reunion of an electron with a positively charged molecule, in which cases the emission centre would be neutral. Luminous vapours emitting band spectra usually appear to be neutral at the instant of emission, so that it seems probable that band emission is due either to elastic shock or to the

1 Address delivered in Section B-Physics-of the American Association for the Advancement of Science at the New York meeting, December, $x x^{16} 6$, by the chairman of the Section, Prof. E. P. Lewis. Continued frem p. 118.

No. 2476, vOL. 99] 
recovery of a lost electron. It is to be remarked that as a rule band spectra are not subject to the Zeeman, Stark or Humphreys-Mohler effect; in the exceptional cases it is probable that those subject to one of these effects are subject to all. It would be of interest to examine these cases with reference to the nature of the molecular charge.

Luminous vapours emitting line spectra appear, in many cases at least, to be positively charged. A sodium flame is attracted to the negative plate of a ccndenser. A metallic salt introduced near the cathode of a spark discharge colours the spark only in that neighbourhood; if introduced near the anode, the colour flashes entirely across the spark. The most promising method of verifying such conclusions appears to be by the study of canal or positive rays. Sir Joseph Thomson, from a study of the deflections produced by magnetic and electric fields, found that, with very few exceptions, no molecules of either elements or compounds carry a negative charge, while those with positive charges are common. No molecule acquires more than one positive charge. The atoms of but few elements are found with a negative charge, but all may acquire positive charges and many may be multiply charged. For example, krypton may have as many as five and mercury eight positive charges. Hydrogen never has more than one charge, which acacrds with Bohr's view that it has but one detachable electron.

Stark has reached similar conclusions from a study of the spectra of canal rays. In many cases the motion in the line of sight gives a Doppler effect. There is an undisplaced line due to the stationary gas and a displaced line due to the canal rays. A distinct sepanation between the displaced and stationary lines shows that the canal rays cannot radiate until their kinetic energy reaches a threshold value, which Stark first interpreted in favour of the quantum theory, but which he now believes to represent the energy necessary for ionisation. There may be two or even three displaced lines, with separations consistent with the view that the luminous centres are doubly or triply charged. The radiation is evidently due to collisions, for a reduction of pressure in the canal ray chamber causes a reduction of luminosity. In general, all series lines are subject to the Doppler effect. Fulcher has shown that nitrogen canal ravs give the negative pole band spectrum, with displacements, but no other bands have been found to give this effect. The series lines of hydrogen show displacements, but they are not observed in the many-line spectrum except to a slight extent in a few cases. Stark concludes that the series lines are emitted by positive atom ions, and the lines of the secondary spectrum by neutral atoms. $\mathrm{He}$ thus assoaiates the compound spectrum with band spectra, which he supposes to be due to neutral systems. It mav be remarked that Fabry and Buisson have concluded from measurements of the width of lines that both snectra are due to emission centres of atomic size. From a study of the displaced components of manv elements, electronegative as well as electropositive, Stark concluded that in all cases line spectra are emitted by positively charged atoms. Aluminium atom ions may have one, two, or three charges, which appear in succession as the voltage is inoreased. The same is true of argon. The red spectrum is apnarently due to singly charged ions, the blue or sparl spectrum to multiple charges. Mercury may have as many as four charges, each giving rise to a characteristic group of lines, all those due to multiple charges being spark lines. From an examination of many such cases Stark concludes that in general arc lines or those of the positive column are due to singly charged ions, sharp spark lines to NO. 2476, VOL. 99] double charges, and diffuse spark lines to triple charges. There are some apparent exceptions to this classification, but in the main the evidence seems to support his views, which are also consistent with the results obtained by Reichenheim from the study of anode rays. For the first time we are thus enabled to assign a common cause for spark lines produced under apparently very different conditions. 'They are found in the spectra of disruptive discharges, of the negative glow in vacuum tubes; in the intermittent or oscillating arc when rapid changes in potential occur, although the maximum potential may be small; near the poles of the arc, where the anode and cathode potential gradients are steep; in the electric furnace when the temperature is high; in high temperature stars, and, as found by Hemsalech and de Watteville, even in tne green cone of the Bunsen flame, where chemical action is energetic. In all these cases we might expect multiple ionisation to be favoured.

Similar conclusions regarding the charges of emission centres may be derived from observations by Stark, Child, Strutt, and others on the luminous vapours from an arc between charged condenser plates. The carriers of the line spectra are swept out of the field, while the luminous vapours giving band spectra are unaffected; or, if the lines of several series are present, their intensities are modified in different degrees by the electric field. Studies of the oscillatory spark by Schuster and Hemsalech, Schenck, Milner, Royds, and others indicate that the spark lines do not persist as long as arc lines. If the emission centres of the former are multiply charged this is what we might expect.

Investigations on the mechanism of the spark give results which at first sight seem opposed to Stark's theory. All observers agree that the luminous vapours appear to be projected from the cathode, with different velocities for different lines, and the tacit assumption seems to have been made that they are negatively charged. That metallic vapours are projected from the cathode is evident from the fact of cathode disintegration, and probably the particles are initially negatively charged. We know very little concerning this phenomenon, but two things are almost certainthat only a small fraction of the metallic particles take part in the luminosity, and that these particles are not negatively charged while radiating. The large velocities indicated by the curvature of the streamers viewed in a rotating mirror do not give rise to a corresponding Doppler effect, and it seems highly probable that Hull and Royds are correct in their surmise that what happens is really the propagation of a condition of luminosity through vapour which continuously fills the gap after the first discharge. Electrons initially projected with a high velocity, which diminishes as the field intensity drops to zero, and producing multiply charged ions in the beginning and singlv charged ions towards the end of their course, would apparently account for all the observed effects.

While the experimental evidence seems to favour the idea that lines are emitted by positively charged centres, there is no a priori reason why neutral or even negative ions should not emit line spectra. It is quite possible that the canal ray lines which Stark attributes to singly charged ions may be emitted at the instant of neutralisation; but we cannot escape the conclusion that spark lines at least are emitted by positive ions unless we accept the improbable view that a multiple charge may be instantaneously entirely neutralised. Lenard inferred from the distribution of emission centres in the arc that the lines of the principal series are emitted by neutral atoms, those 
of subordinate series and spark lines by multiply charged atoms. Wien and others have suggested that line spectra may be emitted by molecules, but this seems improbable. On the other hand, we must admit the possibility of negatively charged centres which would probably exist only under exceptional conditions. Nicholson has, with success, assumed the existence of positive, neutral, and negative centres in accounting for the spectrum of the corona.

The fundamental importance of reaching definite conclusions as to the magnitude of the electric charge of emission centres is evident when we remember that any theory must take this into account. Bohr's theory rests upon the assumption that series lines are emitted by electrons previously detached as they return to equilibrium positions determined by the resultant charge of the system. In the case of hydrogen, if there be but one detachable electron, the radiating system must be neutral. If it can be shown without question that the emission centres of the Balmer series are positively charged, some modification of the theory seems necessary. Furthermore, if the centres are thus deprived of the one detachable electron, we must accept Stark's view that the series emission is due to electrons which cannot be detached. Fulcher has pointed out the necessity for a similar conclusion with respect to helium. Some of its lines are attributed to doubly charged atoms; but these are identical with alpha particles, the nuclei of the atoms, from which the radiation must be emitted.

Beyond the probable fact that band spectra are usually emitted by neutral systems, there is little evidence upon which we may rest a theory. Emission may accompany the neutralisation of a positively charged molecule by an electron or may be the result of internal vibrations due to collisions, without complete ionisation. Stark believes that the band emission is due to the detachable valency electrons, although the coupling between them and more firmly bound electrons may cause the latter to take part.

Evidence supporting Stark's views is to be found in absorption spectra. Hydrogen shows no absorption until it is ionised by a current. The cold vapours of the alkali metals and of mercury show line absorption, but their susoeptibility to the photoelectric effect indicates how ionisation may be the prelude to absorption. All the corresponding emission lines appear to be due to singly charged emission centres. Absorption of the lines due to multiple charges does not take place until the vapour is highly ionised by electric discharges or high temperature. Substances which show band absorption under ordinary conditions, such as iodine, do not appear to be ionised when either emitting or absorbing. Both processes appear to be due to neutral systems. In such cases emission must be due to internal disturbances, without ionisation. The bands of some substances, such as nitrogen, are not found in absorption under any conditions, and the conditions of their occurrence indicate that the emission bands are due to the recombination of a detached electron with a positive molecule. The negative pole bands appear under the same conditions as spark lines, and it seems not improbable that they are due to the neutralisation of a doubly charged molecule.

The spectral differences attending different stages of ionisation are well illustrated by some recent experiments. Franck and Hertz found that mercury vapour is ionised by a field of 4.9 volts, and then emits the one ultra-violet line 2537. The Einstein relation $\mathrm{V} e=h v$ is fulfilled. McLennan and Henderson verified this conclusion, and also found that with a field of about 12 volts a second stage of ionisation occurs, attended by the emission of the many-lined spectrum attributed by Stark to multiple charges. McLennan finds that zinc, cadmium, and magnesium also give single line spectra which probably conform with Einstein's equation, which we should not expect to apply in a simple form to the many-line spectrum.

It appears from such experiments that there is a threshold value of kinetic energy which must be imparted to an emission centre before it can radiate, which represents the work of ionisation and is equal to a light quantum. Franck holds that this energy may be devoted either to ionisation or to emission, but that both cannot simultaneously occur. Stark believes that the two are coincident, the emission accompanying the rearrangement of electrons. in the atom after one has been ejected. This suggests an explanation of quantum emission involving no departure from accepted electromagnetic theory.

The spectra of hydrogen and of helium are of particular interest because their atoms are of the simplest type and because it is possible that they are the basic units of which all elements are composed. The Pickering series in stellar spectra was attributed to hydrogen because of its numerical relationships with the Balmer series. The study of series relations led Rydberg to predict the occurrence of a principal series for hydrogen beginning at wavelength 4686 , and this line was subsequently found in nebular and stellar spectra. After many attempts to reproduce these spectra in the laboratory, Fowler succeeded in $18 \mathrm{I} 2$, by passing a powerful disruptive discharge through a mixture of hydrogen and helium. Produced only under such conditions, these must be classed as spark lines; and if Stark's views are correct and if they are really due to hydrogen, that element must have more than one detachable electron.

In applying his theory to the helium spectrum, and assuming one electron returning to a helium atom from which two electrons have been detached, Bohr obtained a formula which gives lines corresponding in position to those of the Pickering and Rydberg series, and also another series almost coincident with the Balmer hydrogen series. This remarkable conclusion was strengthened by Stark's discovery of 4686 in a helium tube which gave no lines of the ordinary hydrogen spectrum. He concluded from the canairay displacements that the emission centres were doubly charged. Evans also found the first members of all the series assigned to helium by Bohr, including that corresponding to the Balmer series, in a tube containing no hvdrogen. The experimental evidence thus favours Bohr's theory, but we must remember the remarkable way in which the presence of one element mav intensify or suppress the spectrum of another. For example, Lyman found that the ultra-violet series attributed without question to hydrogen is greatly intensified by the presence of helium. It may be added that Merton has con. cluded, from a study of the width of 4686 , that it is due to an atom smaller than that of helium.

Some light may be thrown on this problem by observations such as those made by Wright and others on the distribution of materials in nebulæ, as indi. cated by the length of the nebular lines. Wright finds that usually 4686 is confined to the nucleus; helium lines extend further, and those of nebulum and hydrogen still further. These results favour the view that the elements distribute themselves according to their atomic weights, and that $\Delta 686$ is due to an atom at least as heavy as that of helium. But this is not conclusive, because a high temperature line of hydrogen might be found only in the hot nucleus, if we grant the possibility of a higher degree of ionisation for hydrogen.

Fundamental questions which are of importance to

No. 2476 , voL. 99] 
physicists and astronomers alike are involved in this problem, but it is evidently an elusive one. Curiously enough, as Fowler has proved by comparison with other spectra, general series relations would permit us to assign the disputed series to hydrogen or to helium impartially, and it seems possible that both elements may give the same spectrum under appropriate conditions. Bohr has also concluded from the formula derived from the assumption of the return of an electron to a lithium atom whioh has lost three electrons, that lithium would emit lines close to the Balmer series. Bohr has not yet succeeded in applying his method to the case where an electron returns to a singly charged helium or lithium atom, and hence has not been able to account for the known helium lines, which are assigned by Starls to singly charged atoms. Nor has he taken account of atomic magnetic fields, which, as Humphreys, Allen, and others have shown, may exercise an appreciable influence.

One of the most fascinating fields of research is that of fluorescence and resonance spectra, in which much work has recently been done, particularly by Wood. He has found that white light will excite the complete band and line resonance spectrum of sodium or iodine, but that a single exciting line will cause the emission of a line of the same length, and also of a number of lines approximately equally spaced which may not always coincide in position with one of the absorption lines. Thus the vapour is caused to emit forced vibration, giving a spectrum not its own. As Wood has suggested, this method enables us to strike one key of the complex vibrating system of the atom, instead of the whole keyboard at once. Time does not permit a detailed account of this remarkable work, but it is evident that it may render great service in the study of the mechanism of the atom. Nor is there time even to mention any of the results obtained in the field of absorption spectra.

After reviewing the work of the past decade, we may feel encouraged by the progress that has been made both in the perfecting and application of spectroscopic methods of research and in the discovery of new phenomena. Some of these discoveries have led to fundamental revisions of our sotions of atomic structure. The Rutherford atom has definitely displaced that of Thomson. In some respects this has seemed to make the problem more difficult, but it has at least defined it more precisely. Many attempts have been made to represent an atomic structure which would satisfy the necessary mathematical conditions, most of them so impossible as to be absurd or so speculative that they suggest no experimental tests of their validity. The great merit of Bohr's hypothesis is that it does lend itself to such tests, and it is for that reason that I have paid special attention to the methods of experimental attack which seem to give the most concrete results in this connection. Hesitant as we may be to accept in all its details a theory which asks us to abandon laws upon which we have pinned our faith, this theory, and the quantum theory as well, mav be the flashes of genius which reveal incompletely the outlines of the truth towards which we struggle along a dimly lighted path. Fuller knowledge may resolve some of our difficulties and reconcile apparent contradictions. Ptolemy's theory of epicvcles would appear wholly irrational to one acquainted with Newton's laws but ignorant of Kepler's conclusions, yet it correctly described the facts as Ptolemy saw them. Some day the Kevler and the Newton of the atom mav appear, but their task will not be an easv one. If the astronomer is baffled by the problem of three bodies which he can see, how can we expect to define the exact laws determining the motions of the invisible hosts of No. 2476 , VOL. 99] electrons and positive charges in an atomic system? How can we hope to picture correctly the mechanism which emits radiations of almost infinite complexity, or account for the additional complications called forth by external forces? We may be almost tempted to accept the pessimistic view expressed by Planck in his Columbia lectures, that nothing in the world entitles us to believe that it will ever be possible to represent completely through physical formulæ the inner structure of the atom. And Kayser has said: "A true theory must assume a complete knowledge of electrical and optical processes, and therefore is a Utopia.'

But even if we never reach the goal, who can set a limit to our approach to it? We may never set foot upon the promised land, but some day we may perceive its shadowy outlines dimly from afar.

\section{UNIVERSITY AND EDUCATIONAL INTELLIGENCE.}

ThE Joint Matriculation Board of the Universities of Manchester, Liverpool, Leeds, Sheffield, and Birmingham has revised its regulations for entrance to the faculties of medicine, and no longer requires that Latin should be taken as an obligatory subject. The conditions imposed by the board for entry to the faculty of medicine are now identical with the general conditions for entrance to the several faculties of the Universities.

WE learn from Science that the library of the late Prof. Hugo Münsterberg has been given to Harvard University by a group of his friends. The library consists of about 10,000 books, pamphlets, manuscripts, charts, and other papers. Among the 3000 books in the collection are the latest and most valuable on experimental and applied psychology, especially those bearing on aspects of the subject to which Prof. Münsterberg had devoted his time.

According to a recent article in the Frankfurter Zeitung and an interview with a prominent librarian at Frankfort plans are being considered for the establishment of a greneral technical library at Frankforton-Main, to be open for public use. One of the leading city libraries has become interested in the project, and a beginning has already been made. A demand exists for a library which will be of service to all the numerous branches of the industry and trade in and about Frankfort, the most important industrial centre in South and West Germany. In this manner a broader spirit of scientific and technical investigation will be fostered. An atiemp* will be made to furnish technical information which will have a historical as well as a purely scientific value. Technical libraries have existed previously, but they have not been open to the general public. Such libraries have been the property of scientific societies, technical associations, and the larger industrial concerns. The service rendered by these scattered collections was comparatively small, as it was limited to members of the respective organisations owning them. These were usually hampered by lack of means and lack of facilities for organising and arranging to the best advantage. The plan that is now under way would combine these private and semi-private libraries and put them under the control of one of the established city libraries at Frankfort-on-Main. The library chosen for the purpose is the Frieherrliche Carl von Rothschildsche Offentliche Bibliothel. In addition to technical books, it is stated that the chief technical magazines of Germany and of the world are to be placed at the disposal of the nublic. A special feature will be the department for patent publications. Not only will 